\title{
Leadership Autonomy Support and employee performance: Mediating effect of Intrinsic Motivation
}

\author{
Samsudin Wahab ${ }^{1}$, Adi Izhar Che Ani ${ }^{2}$, Siti Nur Fadzilah Muhsain ${ }^{3}$, Ahmad Rashidy Razali ${ }^{4}$, \\ Nor Aziyah Bakhari ${ }^{5}$ \\ \{ samsudinw@uitm.edu.my ${ }^{1}$, adiizhar@uitm.edu.my ${ }^{2}$, sitinurfadzilah077@uitm.edu.my ${ }^{3}$, \\ ahmad073@uitm.edu.my ${ }^{4}$, noraziyah140@uitm.edu.my ${ }^{5}$ \} \\ Universiti Teknologi MARA, Cawangan Pulau Pinang, Malaysia ${ }^{1}$, Universiti Teknologi MARA, \\ Cawangan Pulau Pinang, Malaysia ${ }^{2}$, Universiti Teknologi MARA, Cawangan Pulau Pinang, Malaysia ${ }^{3}$, \\ Universiti Teknologi MARA, Cawangan Pulau Pinang, Malaysia ${ }^{4}$, Universiti Teknologi MARA, \\ Cawangan Pulau Pinang, Malaysia ${ }^{5}$
}

\begin{abstract}
The focal objective of this research is to examine the empirical relationship between Leadership Autonomy Support (LAS), Employees Well-being and Performance while considering intrinsic motivation and workplace climate as a mediator construct in relation to the proposed theoretical framework. This study will significantly extend the existing literature by considering the Pakistan's cultural context in relation to the Leader Autonomy Support (LAS), Employees Personal Well-being and productivity by applying SDT (Self-Determination Theory). In addition, the current empirical evidence will also exhibit the focal implications for the academia and industry practitioners.
\end{abstract}

Keywords: Leadership Autonomy Support (LAS), Employee Well-Being, Workplace Climate, Employee Performance.

\section{Introduction}

Since the industrial revolution, there has been increasing attention towards how leaders can engender and sustain motivation and human capital performance at the workplace. Based on the several theories of leadership, more specifically, Self-Determination Theory (SDT), we may argue that Leader Autonomy Support (LAS) is a leadership style which is the stronger predictor of employee's internal motivational resources, LAS is defined by leaders who exhibit interest towards their employee's perspective, ensure the provision of input and choice opportunities, engender self-esteem and resilience, and refrain from the use of negative reinforcements aiming to attain desired behaviors, which consequently, optimize the employee's level of engagement, well-being and productivity in the organizational work environment [1] [2].

Past studies exhibit mixed findings in relation to the effects of LAS on organizational personnel well-being and performance, clarity is required in relation to the theoretical framework involved. Consequently, The focal objective of this research is to examine the empirical relationship between LAS, Employee Personal Well-being and Performance through the mediating role of Workplace Climate and Intrinsic Motivation by adopting SDT Framework. The focal emphasis of the SDT theory towards people's volitional motivation which is described as the extent to which they perceived their actions as autonomous (for 
instance, actions based on values, happiness, interest and choice) or controlled (for instance, actions based on obligations, feelings of guilt, punishment and rewards). Based on the various empirical evidences generated on the basis of SDT perspective, we may argue that autonomous kinds of motivation are seems to be aligned with the more constructive organizational outcomes, for instance, Job satisfaction, persistence, commitment and selfregulation and the LAS can also trigger autonomous type of motivation among the organizational members (e.g., in [3]). Current research will also shed the light on the empirical summary of the noticed correlations in the existing literature; various theoretical frameworks exhibit the areas of further interest for the future scholars. We initiate our study by documenting a short history and background of LAS in relation to the workplace, and then we provide a fundamental overview regarding the various categories of motivation with reference to the SDT perspective and explain how these constructs are related to the LAS at the workplace. Moreover, we shall also review the potential mediators of effects, and then investigate how LAS significantly contributed towards the employee-centric outcomes, for instance, well-being and performance.

\section{Literature Review}

Despite of various kinds of motivation, for instance, degree of autonomy, the focal emphasis of SDT is towards the significance of personal well-being and supportive autonomy environment. Generally, it has been evident that perceived autonomy support engender the autonomous kind of behavioral regulations among the organizational members. Plenty of research has been documented on the significance of supportive autonomous environment that significantly related to the fostering of personal well-being and autonomous motivation [4] [5] [6] [7].

Although, few researchers demonstrated that how LAS is mainly related to five various types of motivation. Extending past studies, the current empirical evidence will examine the direct and indirect relationship between LAS, well-being and performance by considering workplace climate and intrinsic motivation as mediators constructs. In relation to the various types of motivation, the majority of the researchers categorized motivation into two types, autonomous motivation (the amalgamation of identifies regulation and intrinsic motivation) and controlled motivation (the amalgamation of introjected and external regulation) (e.g., in [3] [5] [8].

Since the industrial revolution, employee motivation and performance is conceptualized as to be driven by LAS provided at the workplace [9]. As a result, plenty of research has been documented that exhibit how LAS is mainly associated with the behaviors and motivation at the workplace. Initial attention to the topic can be traced through the Taylor's work on "scientific management" where the follower's motivation was perceived as a consequence of scientifically crafted reward system. Recently, s few studies have been generated that grab the attention of the contemporary scholars on the notions of intrinsic and extrinsic motivation as a framework of SDT [10]. SDT perspective is wider with reference to the human motivation that is aligned with the individual's instinctive growth dispositions and pivotal cognitive requirements emphasize on the extent to which behavior of the individual is perceived as controlled or autonomously motivated. It has been applied among the various research contexts and industries, for instance, parenting, health, sports, education and organizations (e.g., in [2] [11]). 
With reference to the organizational domain, it has been evident that the contextual elements strongly predict the organizational personnel focal cognitive needs and selfdetermination (e.g., in [12]). Job autonomy has been defined as " the extent to which individual workers can self-govern how and when they perform the various tasks that make up their job", is generally perceived as a contextual antecedent in relation to the selfdetermination at the organization work settings. In relation to the job design at the organizational work settings, we may argue that LAS can be generated on the basis of a personal hallmarks exhibit by the organizational leadership, for instance, leadership styles and patterns of motivation [11] [13]. LAS refer to an array of leadership behaviors that jointly engender the support climate and clarity in relation to the supervisor-subordinates dyads [11]. LAS generally comprised by the various leadership traits which includes employee acknowledgement, fostering self-initiation at the workplace, decentralization and refrain from the negative and positive reinforcements aiming to control behaviors and offering choice and input opportunities to the organizational members [2]. It is essential to conceptualize that fostering a sense of self-determined among the organizational employees, organizational leadership should engender the feelings that behaviors are internally directed rather than externally controlled in an organizational work environment. On the other hand, a controlling leadership style exerts external restrictions on employee's behaviors in order to force organizational members to exhibit desired behaviors. A controlling leadership style generally perceived as more rigid, inflexible, authoritarian, pressing employees to feel, think and behave in specific ways. Noncompliance with the leadership requirements triggered disciplinary actions aiming to generate desired behaviors [13].

\subsection{Intrinsic Motivation as a Mediator between LAS \& Well-being}

It has been demonstrated that LAS is an antecedent of several kinds of motivations which are the strongest predictors of employee's well-being at the workplace (e.g., in [6]). It has been suggested in the previous studies that it is reasonable to choose employee's intrinsic motivation as a mediator construct between LAS and well-being. Although, past studies investigated the mediating effects of intrinsic motivation and on LAS and well being in the Chinese context, but empirical evidence is not available in relation to the Pakistan's context. The focal purpose of this research is to fill the void in the existing literature by choosing intrinsic motivation as a mediator construct with reference to the banking sector of Pakistan. In relation to the SDT, we may argue that LAS is a strong predictor of well-being and performance at the workplace, across various cultural contexts based on focal cognitive needs, for instance, autonomy needs [14]. Few studies shown that LAS is significantly contributed only in those cultures in which autonomy is perceived as more valuable. Consequently, the focal question of this empirical research is whether LAS is still beneficial or desirable in a cultural context that may not an exhibit explicit value in relation to the significance of autonomy support. Particularly, the current empirical evidence extends the available literature by examining the direct relationship between LAS, well-being and performance and considering intrinsic motivation and workplace climate as mediators constructs in relation to the proposed theoretical framework in a Pakistan's cultural context by using a sample of the banking sector of Pakistan. 


\section{Methods}

In their routine lives, people generally perform deeds of kindness or do small favors for others. Few of these kind deeds are independently chosen and from the core of heart, for instance, purchasing a coffee cup for a friend. It has been evident that the pro-social attitudes and behaviors significantly associated with the employees personal well-being when autonomy support provided in the organizational work settings [15]. Yet, little is known about whether and how it might be possible to engender autonomy in the context of everyday kindnesses.

The current study shall have the three focal objectives. First, we will examine the role of autonomy support in relation with the employee personal well-being and performance. Second, we will tap the mediating role of intrinsic motivation and workplace climate between autonomy support, personal well-being and performance. Finally, the third focal purpose of this empirical investigation is to test the generalizability of our findings across Pakistan's culture. Autonomy support provided in the organizations is mainly related to the increasing level of personal well-being across various cultures, this correlation may be exist on the basis of psychological universal [16] [17]. Autonomy support can be executed effectively among several nations. To examine whether the focal mechanisms of the suggested link between autonomy support, well-being and performance are identical across cultures, data shall be collected from the banking sector of Pakistan. The proposed framework for this study is shown in Figure 1.

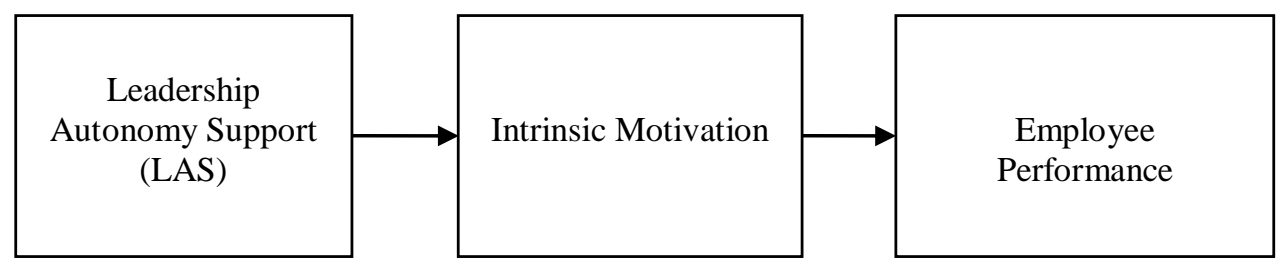

Figure 1: Proposed Framework

\section{Result and Discussion}

Very big issues on leadership is the feedback subordinate to listen the order by their leader. Most leader failed to motivated their subordinate as a result their team not really committed to the task. In nursing routine job, most of them facing real tension and stress due to numbers of patient arising from time to time. especially in the corona virus phenomenon, part of them are front liners that involved directly to the situation. LAS consequently influence subordinate motivation, then directly linked to employee performance. Previous study confirmed the relationship between this three variables. 


\section{Conclusion}

As a conclusion, the banking sector must open their eyes on the routine activities in the counter. Clerical staff performance are important to make sure high quality of services offered to the customer. Both parties important role in achieving high productivity in term of ROI and non-financial performance.

\section{Acknowledgements}

Acknowledgemets. Special appreciation to Universiti Teknologi MARA, Cawangan Pulau Pinang for providing the budget for this research study.

\section{References}

[1] M. Vansteenkiste, W. Lens, and E. L. Deci, "Intrinsic Versus Extrinsic Goal Contents in SelfDetermination," Educ. Psychol., 2006.

[2] Y. L. Su and J. Reeve, "A Meta-analysis of the Effectiveness of Intervention Programs Designed to Support Autonomy," Educational Psychology Review. 2011, doi: 10.1007/s10648010-9142-7.

[3] C. Fernet, S. Austin, S. G. Trépanier, and M. Dussault, "How do job characteristics contribute to burnout? Exploring the distinct mediating roles of perceived autonomy, competence, and relatedness," Eur. J. Work Organ. Psychol., 2013, doi: 10.1080/1359432X.2011.632161.

[4] V. I. Chirkov, "A cross-cultural analysis of autonomy in education: A self-determination theory perspective," Theory Res. Educ., 2009, doi: 10.1177/1477878509104330.

[5] E. Kins, W. Beyers, B. Soenens, and M. Vansteenkiste, "Patterns of Home Leaving and Subjective Well-Being in Emerging Adulthood: The Role of Motivational Processes and Parental Autonomy Support," Dev. Psychol., 2009, doi: 10.1037/a0015580.

[6] L. G. Pelletier, M. S. Fortier, R. J. Vallerand, and N. M. Brière, "Associations among perceived autonomy support, forms of self-regulation, and persistence: A prospective study," Motiv. Emot., 2001, doi: 10.1023/A:1014805132406.

[7] I. M. Taylor and N. Ntoumanis, "Teacher Motivational Strategies and Student SelfDetermination in Physical Education," J. Educ. Psychol., 2007, doi: 10.1037/00220663.99.4.747.

[8] M. Vansteenkiste, M. Zhou, W. Lens, and B. Soenens, "Experiences of autonomy and control among Chinese learners: Vitalizing or immobilizing?," J. Educ. Psychol., 2005, doi: 10.1037/0022-0663.97.3.468.

[9] M. H. Gilbert, V. Dagenais-Desmarais, and F. St-Hilaire, "Transformational leadership and autonomy support management behaviors: The role of specificity in predicting employees' psychological health,” Leadersh. Organ. Dev. J., 2017, doi: 10.1108/LODJ-08-2015-0173.

[10] E. L. Deci and R. M. Ryan, "Facilitating optimal motivation and psychological well-being across life's domains': Correction to Deci and Ryan (2008).," Can. Psychol. Can., 2008, doi: 10.1037/0708-5591.49.3.262.

[11] S. H. Cheon, J. Reeve, J. Lee, and Y. Lee, "Giving and receiving autonomy support in a highstakes sport context: A field-based experiment during the 2012 London Paralympic Games," Psychol. Sport Exerc., 2015, doi: 10.1016/j.psychsport.2015.02.007.

[12] V. Den Broeck, M. Vansteenkiste, and H. De Witte, "Self-determination theory: A Theoretical and Empirical Overview In Occupational Health Psychology," ... Theor. Soc. Psychol., 2011.

[13] E. L. Deci, J. P. Connell, and R. M. Ryan, "Self-Determination in a Work Organization," J. 
Appl. Psychol., 1989, doi: 10.1037/0021-9010.74.4.580.

[14] S. N. Chua, N. Wong, and R. Koestner, "Autonomy and controlling support are two sides of the same coin," Pers. Individ. Dif., 2014, doi: 10.1016/j.paid.2014.04.008.

[15] N. Weinstein and R. M. Ryan, "When Helping Helps: Autonomous Motivation for Prosocial Behavior and Its Influence on Well-Being for the Helper and Recipient," J. Pers. Soc. Psychol., 2010, doi: 10.1037/a0016984.

[16] L. B. Aknin et al., "Prosocial spending and well-being: Cross-cultural evidence for a psychological universal,” J. Pers. Soc. Psychol., 2013, doi: 10.1037/a0031578.

[17] S. Lyubomirsky and K. Layous, "How Do Simple Positive Activities Increase Well-Being?," Curr. Dir. Psychol. Sci., 2013, doi: 10.1177/0963721412469809. 